\title{
Long-Term Clinical Course of Asthma Recorded by Digital Asthma Diary: A Case Report
}

\section{Gon $\mathbf{Y}^{\star}$, Maruoka S, Mizumura K, Ito R, Kumasawa F and Hashimoto S}

Division of Respiratory Medicine, Department of Internal Medicine, Niohn University School of Medicine, Tokyo, Japan

"Corresponding author: Gon Y, Division of Respiratory Medicine, Department of Internal Medicine, Niohn University School of Medicine, Tokyo, Japan, Tel: +81-3-3972-8111; Fax: +81-3-3972-2893; e-mail: gon.yasuhiro@nihon-u.ac.jp

Received date: April 19, 2017; Accepted date: April 29, 2017; Published date: May 5, 2017

Copyright: ( 2017 Gon Y, et al. This is an open-access article distributed under the terms of the Creative Commons Attribution License, which permits unrestricted use, distribution, and reproduction in any medium, provided the original author and source are credited.

\begin{abstract}
Self-management methods for chronic diseases using mobile phone and tablet applications have recently become popular. Although several applications for asthma management are available, there are no reports concerning the long-term treatment effects of inhaled corticosteroid (ICS)/long-acting beta agonist (LABA) treatment on diurnal peak flow variability according to digital asthma diary records or its utility. A 31-year-old female diagnosed with moderate persistent asthma was treated with ICS/LABA. On approximately 2 years after the treatment, we were able to observe that diurnal peak flow variability disappeared gradually and completely when the time axis set that all records can be observed in one screen. Self-monitoring the peak flow using digital asthma diaries is suitable for long-term recording and allows an adequate understanding of long-term asthma state and treatment effects especially for minor diurnal peak flow variability by ICS/LABA.
\end{abstract}

Keywords: Asthma; Diurnal peak flow variability; Digital asthma diary; Airway hyper responsiveness

List of Abbreviations: GINA: Global Initiative for Asthma; FeNo: Fractional Exhaled Nitric Oxide; LABA: Long-Acting Beta Agonist; ICS: Inhaled Corticosteroid; ACT: Asthma Control Test

\section{Introduction}

Technological advances and the widespread use of mobile devices have led to their use in healthcare management. The use of such healthcare management methods is expected to have a major impact on the management of chronic diseases.

Bronchial asthma is a chronic inflammatory airway disorder that presents with symptoms related to reversible airway constriction. The long-term use of inhaled steroids is required for well control of asthma. As recommended in the 2010 Global Initiative for Asthma (GINA) incorporating guided self-management into asthma treatment lowers the mortality rate of the disease [1-4]. The objective evaluation of the control level of asthma requires that the patients themselves use a portable peak flow meter to measure peak flow values. This offers the advantage of allowing their physician to view these records based on which subsequent treatment and guidance can be planned. Consequently, the patient understands the necessity of visiting hospital, and reliably follows instructions regarding behaviors aimed at avoiding exacerbation [2-4]. Although several applications for asthma management are available there are no reports concerning the longterm treatment effects of inhaled corticosteroid (ICS)/long-acting beta agonist (LABA) treatment on diurnal peak flow variability according to digital asthma diary records or its utility.

We experienced a case with asthma who has recorded the peak flow for long-term using digital asthma diary. We reaffirmed utility of digital diary for an adequate understanding of long-term asthma state and treatment effects.

\section{Case Report}

Patient: 31 year old woman

Chief complaint: Discomfort in the chest region during respiration

Medical history: 28 years, atopic dermatitis, childhood asthma

Family history: Nothing in particular

Patient: 31-year-old woman

Chief complaint: Discomfort in the chest region during respiration

Medical history: 28 years, atopic dermatitis, childhood asthma

Family history: Nothing in particular

\section{Physical findings at initial examination were as follows:}

Blood test results at initial examination: WBC- 4,600/ $\mu$ l (eosino.

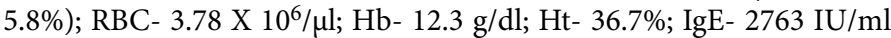
(Dermatophagoides pteronyssinus $6+$, Dermatophagoides farinae $6+$, Aspergillus+).

\section{Chest X-ray: No abnormalities}

Pulmonary function test: FVC-3.62 L, \%FVC-118\%, $\mathrm{FEV}_{1}-3.13$, $\% \mathrm{FEV}_{1}, \mathrm{PFE}-6.66 \mathrm{~L} / \mathrm{sec}, \mathrm{FEV}_{1} \% 89 \%$.

Course: Her asthma control test (ACT) score was 17 points. Fractional exhaled nitric oxide (FeNO) was low at $11 \mathrm{bpp}$, therefore, the patient was diagnosed moderate persistent asthma. She was administered $640 \mu \mathrm{g} / 18 \mu \mathrm{g}$ of budesonide/formoterol and oral administration of montelukast sodium was initiated. Currently, 1 year and 10 months after treatment was first initiated; the patient has been stepped-down to a low-dose ICS single treatment. Her symptoms have stabilized, ACT score is 25 points. She has measured morning and evening peak flow daily without fail for 1 year and 10 months. She input these values into a digital asthma diary using her mobile phone. Below is a graph showing long-term changes in diurnal peak flow 
variability with long-term inhalation therapy, with the time axis set so that all records in one screen can be observed (Figure 1).

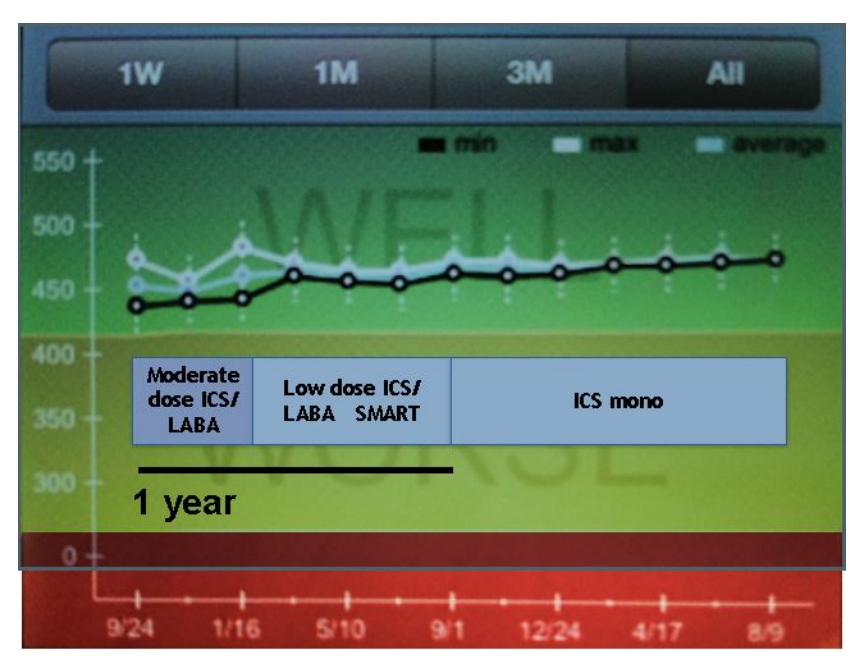

Figure 1: Long-term recording of peak flow using a digital asthma diary, peak flow recorded over 1 year and 10 months using a digital asthma diary. The white line indicates the maximal peak flow value (night peak flow value), the black line indicates the minimal peak flow value (morning peak flow value), and the blue line indicates the mean peak flow value over 1 day.

Improvements with treatment over approximately 6 months in diurnal peak flow variability of less than $10 \%$ can be observed and furthermore, over the next year, it can also be observed that diurnal peak flow variability disappeared gradually and completely. In addition, the peak flow values were increased during the period despite the dose of ICS was reduced after step-down.

\section{Discussion}

Woolcock AJ reported that improvements in symptoms, pulmonary function and airway hyper responsiveness were brought about by inhaled steroids in order of improvements in the night-time symptoms, $\mathrm{FEV}_{1}$, morning peak flow, frequency of use of short-acting beta 2 agonists and airway hyper responsiveness. In order to improve airway hyper responsiveness in particular long-term treatment is required [5]. A real-life study comparatively investigated asthma control state over 3 years in three groups that were administered Adoair 250, Flutide 250 $\mu \mathrm{g}$, or Serevent $50 \mu \mathrm{g}$ while making necessary dosage adjustments during routine medical care. A randomized double-blinded study was conducted in the first year and an open study was conducted in the following 2 years. The analysis of the whole 3 years of observation indicated that symptom scores and pulmonary function improved relatively quickly, whereas airway hyper responsiveness improved gradually over the 3 years [6]. Indeed, in our patient diurnal peak flow variability disappeared over approximately 6 months which may be related to the improvement in airway hyper responsiveness brought about by the long-term inhalation of steroids.

In general, long-term inhalation therapy requires a sufficient amount of patient education including the establishment of therapeutic goals, the provision of a sufficient amount of information, information regarding self-assessment methods and coping strategies that utilize self-management. A recent study has shown that more satisfactory therapeutic effects are achieved and adherence is improved when there is patient participation in the process of determining a therapeutic course, rather than when the physician simply informs a patient of the therapeutic strategy [7]. Proactive patient participation in the process of creating an asthma therapy plan is an effective means of solving the problem of patient non-compliance to treatment plans.

In recent years, with the spread of smart phones and tablets, the methods of self-management for chronic diseases using this technology has come under the spotlight. Several applications for asthma management have also become available including digital asthma diaries in which peak flow values and symptoms can be recorded [8-11]. Conventional asthma diaries, on which data are recorded on paper book, are unsuitable for viewing long-term recording that would allow grasping overall trends. However, digital asthma diaries are suitable for long-term recording and involve the use of portable devices, such as cell phones. In addition, when analyzing records, they can be examined on various time-axis scales and thus are excellent for understanding long-term asthma state and treatment effects [12,13]. Here, we present a case of an asthma patient who recorded her morning and evening peak flow values in a digital asthma diary using her mobile phone for 1 year and 10 months from the initiation of treatment. A correlation between airway hyper responsiveness and diurnal peak flow variability has been reported $[12,13]$. Improvement of peak flow variability to less than $10 \%$ is considered to be a marker signifying asthma control [14]. Using this digital asthma diary, we were able to observe the improvement process of diurnal peak flow variability within $10 \%$ during the long-term treatment.

Another important point is that the patient indicated that continuous record-keeping using the digital asthma diary motivated her to continue asthma treatment and allowed her to get a real, palpable sense of the benefit of continuous inhalation therapy.

\section{Conclusions}

We were able to visually grasp the long-term treatment effects on diurnal peak flow variability in an asthma patient who recorded morning and evening peak flow values in a digital asthma diary for 1 year and 10 months after the initiation of treatment.

The use of a digital asthma diary was suitable for observing longterm improvements in peak flow. The digital asthma diary allowed for a level of information sharing that was previously impossible with the use of paper diaries. The use of this type of asthma diary as a way to share information with the patient regarding their therapeutic progress is effective for improving patient adherence to inhalation therapy and for creating appropriate treatment plans. Therefore, we believe that patient monitoring using digital asthma diaries may be effective for asthma management.

\section{Conflict of Interest}

YG received honoraria from Nippon Boehringer Ingelheim, AstraZeneca and Novartis Pharmaceuticals. SH received honoraria from Nippon Boehringer Ingelheim, GlaxoSmithKline, Kyorin Pharmaceutical, AstraZeneca and Novartis Pharmaceuticals. YG, SM and $\mathrm{SH}$ received research funding from Nippon Boehringer Ingelheim and Kyorin Pharmaceutical. Other authors have no conflict of interest. 
Citation: Gon Y, Maruoka S, Mizumura K, Ito R, Kumasawa F, et al. (2017) Long-Term Clinical Course of Asthma Recorded by Digital Asthma Diary: A Case Report. J Clin Respir Dis Care 3: 125. doi:10.4172/2472-1247.1000125

Page 3 of 3

\section{Acknowledgement}

Yasuhiro Gon, Shuichiro Maruoka, Reiko Ito, Kenji Mizumura wrote the manuscript. Shu Hashimoto and Tomohiro Hatori, Fumio Kumasawa revised the manuscript. All authors approved the final manuscript for publication.

\section{References}

1. GINA (2011) Global Initiative for Asthma: Global strategy for asthma management and prevention.

2. Charlton I, Charlton G, Broomfield J, Mullee MA (1990) Evaluation of peak flow and symptoms only self-management plans for control of asthma in general practice. BMJ 301: 1355-1359.

3. Coté J, Cartier A, Robichaud P, Boutin H, Malo JL, et al. (1997) Influence on asthma morbidity of asthma education programs based on selfmanagement plans following treatment optimization. Am J Respir Crit Care Med 155: 1509-1514.

4. Turner MO, Taylor D, Bennett R, Fitzgerald JM (1998) A randomized trial comparing peak expiratory flow and symptom self-management plans for patients with asthma attending a primary care clinic. Am J Respir Crit Care Med 157: 540-546.

5. Woolcock AJ (2001) What are the important questions in the treatment of asthma? Clin Exp Aller Rev 1: 62-64.

6. Lundback B, Ronmark E, Lindberg A, Jonsson AC, Larsson LG, et al. (2009) Asthma control over 3 years in a real-life study. Respir Med 103: 348-355.
7. Wilson SR, Strub P, Buist AS, Knowles SB, Lavori PW, et al. (2010) Shared treatment decision making improves adherence and outcomes in poorly controlled asthma. Am J Respir Crit Care Med 181: 566-577.

8. Cushing CC, Jensen CD, Steele RG (2011) An evaluation of a personal electronic device to enhance self-monitoring adherence in a pediatric weight management program using a multiple baseline design. J Pediatr Psychol 36: 301-307.

9. Kaiser PK, Wang YZ, He YG, Weisberger A, Wolf S, et al. (2013) Feasibility of a novel remote daily monitoring system for age-related macular degeneration using mobile handheld devices: results of a pilot study. Retina 33: 1863-1870.

10. Albrecht UV, Noll C, Jan UV (2014) App-synopsis: Self-assessment on trust or distrust of health-apps. Stud Health Technol Inform 202: 233-236.

11. Nundy S, Lu CY, Hogan P, Mishra A, Peek ME (2014) Using patientgenerated health data from mobile technologies for diabetes selfmanagement support: Provider perspectives from an academic medical center. J Diabetes Sci Technol 8: 74-82.

12. Godschalk I, Brackel HJ, Peters JC, Bogaard JM (1996) Assessment of accuracy and applicability of a portable electronic diary card spirometer for asthma treatment. Respir Med 90: 619-622.

13. Berg J, Jacob JD, Rohay JM (1998) Compliance with inhaled medications: the relationship between diary and electronic monitor. Ann Behav Med 20: 36-38.

14. Ohta K, Yamaguchi M, Akiyama K, Adachi M, Ichinose M, et al. (2011) Japanese guideline for adult asthma. Allergol Int 60: 115-145. 